\title{
In vitro effect of resin infiltrant on resistance of sound enamel surfaces in permanent teeth to demineralization
}

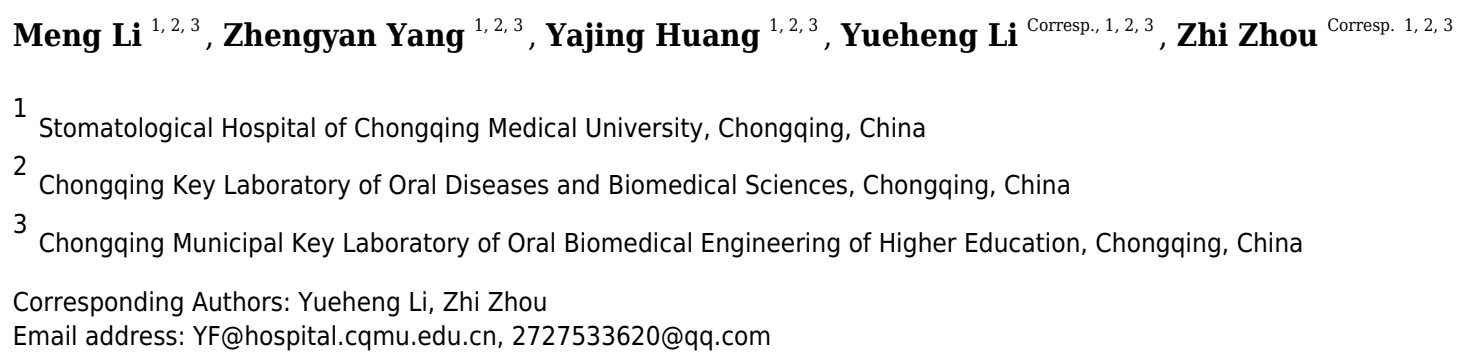

Objective. To investigate the effect of resin infiltrant on resistance of sound permanent enamel surfaces to demineralization. Method. Eighty healthy premolars were sectioned to obtain enamel blocks from the buccal surface. Specimens with baseline surface microhardness values of 320-370 were selected. The experimental group were treated with resin infiltrant, while the control group was not. Specimens from each group were artificially demineralized and the surface microhardness values were measured again. C onfocal laser scanning microscopy was used to measure the depth of demineralization and detect the penetration ability of the resin infiltrant. The specimens were subjected to a simulated toothbrushing abrasion test. Scanning electron microscopy was used to observe changes in the surface morphology of specimens after each of these procedure s.

Results. No significant differences between the experimental and control groups were observed in the baseline microhardness values or in the experimental group after resin infiltration compared with the baseline conditions. After artificial demineralization, the microhardness value in the control group was significantly lower than that in the experimental group (266.0 ( \pm 34.5 ) compared with $304.0( \pm 13.0), P=0.017)$. Confocal laser scanning microscopy results showed that the demineralization depth in the control group was significantly deeper than that in the experimental group (97.9 ( \pm 22.8$) \mu \mathrm{m}$ vs. $50.4( \pm 14.3) \mu \mathrm{m}, P<0.001)$, and that resin infiltrant completely penetrated the acidetched demineralized area of the tooth enamel with a mean penetration depth of 31.6 ( \pm 9.0) $\mu \mathrm{m}$. Scanning electron microscopy showed that the surface morphology was more uniform and smoother after simulated toothbrushing. The enamel surface structure was more severely destroyed in the control group after artificial demineralization compared with that of the experimental group. Conclusion. Resin infiltrant can completely penetrate an acid-etched demineralized enamel area and improve resistance of sound enamel surfaces to demineralization. Our findings provide an experimental basis for 
preventive application of resin infiltrant to sound enamel surfaces to protect tooth enamel against demineralization. 
1 In vitro effect of resin infiltrant on resistance of sound enamel surfaces in permanent teeth

2 to demineralization

3 Meng Li ${ }^{1,2,3}$, Zhengyan Yang ${ }^{1,2,3}$, Yajing Huang ${ }^{1,2,3}$, Yueheng $\operatorname{Li}^{1,2,3}$, Zhi Zhou ${ }^{1,2,3}$

$4{ }^{1}$ Stomatological Hospital of Chongqing Medical University, Chongqing, China.

$5 \quad{ }^{2}$ Chongqing Key Laboratory of Oral Diseases and Biomedical Sciences, Chongqing, China.

$6{ }^{3}$ Chongqing Municipal Key Laboratory of Oral Biomedical Engineering of Higher Education,

7 Chongqing, China.

8

9 Corresponding Author:

10 Yueheng $\mathrm{Li}^{1,2,3}$

11 Email address: YF@hospital.cqmu.edu.cn

12 Zhi Zhou ${ }^{1,2,3}$

13 Email address: 2727533620@qq.com 


\section{Abstract}

15 Objective. To investigate the effect of resin infiltrant on resistance of sound permanent enamel surfaces to demineralization.

17 Method. Eighty healthy premolars were sectioned to obtain enamel blocks from the buccal surface. Specimens with baseline surface microhardness values of 320-370 were selected. The experimental group were treated with resin infiltrant, while the control group was not. Specimens from each group were artificially demineralized and the surface microhardness values were measured again. Confocal laser scanning microscopy was used to measure the depth of demineralization and detect the penetration ability of the resin infiltrant. The specimens were subjected to a simulated toothbrushing abrasion test. Scanning electron microscopy was used to observe changes in the surface morphology of specimens after each of these procedures.

Results. No significant differences between the experimental and control groups were observed in the baseline microhardness values or in the experimental group after resin infiltration compared with the baseline conditions. After artificial demineralization, the microhardness value in the control group was significantly lower than that in the experimental group $(266.0( \pm 34.5)$ compared with $304.0( \pm 13.0), P=0.017)$. Confocal laser scanning microscopy results showed that the demineralization depth in the control group was significantly deeper than that in the experimental group $(97.9( \pm 22.8) \mu \mathrm{m}$ vs. $50.4( \pm 14.3) \mu \mathrm{m}, P<0.001)$, and that resin infiltrant completely penetrated the acid-etched demineralized area of the tooth enamel with a mean penetration depth of $31.6( \pm 9.0) \mu \mathrm{m}$. Scanning electron microscopy showed that the surface morphology was more uniform and smoother after simulated toothbrushing. The enamel surface 
structure was more severely destroyed in the control group after artificial demineralization compared with that of the experimental group.

Conclusion. Resin infiltrant can completely penetrate an acid-etched demineralized enamel area and improve resistance of sound enamel surfaces to demineralization. Our findings provide an experimental basis for preventive application of resin infiltrant to sound enamel surfaces to protect tooth enamel against demineralization.

\section{Introduction}

Resin infiltrant is a low-viscosity (Ammari et al., 2014; Oliveira et al., 2020), light-cured resin with high penetration ability (Paris, Meyer-Lueckel \& Kielbassa, 2007; Kielbassa, Muller \& Gernhardt, 2009). The basic principle of resin infiltration is to penetrate and occlude the porous volume of subsurface lesions by capillary forces, thereby partially or completely replacing the missing minerals, enveloping the hydroxyapatite crystals, micromechanically interlocking the remaining enamel prisms. This method effectively constructs a covalently bonded threedimensional polymer framework (Kielbassa, Muller \& Gernhardt, 2009; Kashbour et al., 2020) and occluding diffusion pathways for cariogenic acids and dissolved minerals to arrest proximal subsurface lesion progress (Meyer-Lueckel et al., 2011; Askar et al., 2018). Previous studies on resin infiltration have mainly focused on its effect on non-cavitated proximal lesions (Araújo et al., 2015; Arthur et al., 2018), enamel white-spot lesions (Markowitz \& Carey, 2018; Silva, et al., 2018; Youssef et al., 2020), fluorosis (Sekundo \& Frese, 2020), and dentin hypersensitivity (Liu Chao et al., 2015). The technique is effective in preventing the progression of initial caries in 
56

57

primary and permanent teeth (Faghihian et al., 2019), and treatment of early dental caries with resin infiltrant achieved excellent clinical results (Lasfargues et al., 2013; Schwendicke et al., 2014; Faghihian et al., 2019; Youssef et al., 2020). Resin infiltration is a non-invasive dental treatment option that complements the concept of minimum intervention dentistry (Lasfargues et al., 2013), and its use is closing the gap between application of oral hygiene and minimally invasive dentistry (Kielbassa, Muller \& Gernhardt, 2009).

Dental caries is the most common chronic oral disease in children and adults worldwide, and is the main cause of defects in dental hard tissue and oral pain, which can seriously affect people's quality of life. In recent decades, the management of dental caries has shifted from drilling and filling to prevention, control, and minimally invasive operative repair in order to preserve more dental tissue (Lasfargues et al., 2013). Fluoride has been widely used for the prevention of dental caries since the mid-20th century (Oh et al., 2017). Dental sealants were introduced in the 1960s to help prevent dental caries, mainly in the pits and fissures of occlusal tooth surfaces. Sealants act to prevent bacterial growth that can lead to dental decay (Faghihian et al., 2019). Research has shown that application of fluoride varnish or resin-based fissure sealants to first permanent molars helps prevent occlusal caries (Kashbour et al., 2020). In general, these preventive measures will be affected by people's compliance, oral hygiene habits, etc. Therefore, it is the unremitting pursuit of the majority of medical workers to continuously find more ways to prevent dental caries.

At present, there is no research on the use of resin infiltrant penetrating agent to prevent oral health. Therefore, in the present study, we explored the possibility of using resin infiltrant for 
77 primary prevention of dental caries. We used resin infiltrant to treat sound enamel surfaces and

performed artificial demineralization, then measured the changes in surface hardness of the enamel and assessed the demineralization depth and degree of penetration. At the same time observe the changes in the surface morphology of samples during the experiment. The effect of resin infiltrant on resistance of tooth enamel to acid erosion and demineralization was explored. The null hypothesis tested was that resin infiltrant can improve the ability of sound enamel surfaces to resist acid erosion and demineralization and completely penetrate an acid-etched, demineralized enamel area.

\section{Materials \& Methods}

The Stomatological Hospital of Chongqing Medical University granted ethical approval to carry out the study within its facilities and participant's written consent has been obtained before tooth extraction. The ethical approval date is May 15, 2018, and the number is CQHS-REC2018(LSNo.22).

Experimental flow chart related to the experimental procedures in this study was shown in Fig.1.

\section{Tooth selection and sample preparation}

A total of eighty premolars from patients who underwent orthodontic treatment involving extraction of premolars in the Department of Maxillofacial Surgery of Stomatological obtained from all subjects prior to sample collection. After removal of roots and soft tissue, the premolars were observed under a fully automatic fluorescence stereomicroscope (Leica M205FA; Leica Microsystems, Baden-wuerttemberg, Germany) to ensure that they were intact 
and contained no cracks or white spots. The teeth were stored in $0.1 \%$ thymol solution (Solarbio;

Solarbio, Beijing, China) until use, which was within one month of extraction. Enamel blocks (4

$\mathrm{mm} \times 4 \mathrm{~mm} \times 2 \mathrm{~mm}$ ) were obtained from the buccal surfaces of the teeth using a hard-tissue

cutting machine (EXAKT 300CP; EXAKT, Hamburg, Germany). The enamel surface was

lightly polished in sequence with \#400, \#800, \#1200, \#2500, and \#5000 silicon carbide abrasive

sandpaper ((MATADOR; Eastern supplier, Remscheid, Germany) under running water to create

a flat surface, leaving an exposed window of $3 \mathrm{~mm} \times 3 \mathrm{~mm}$ in the center of the enamel surface

(Gurdogan, Ozdemir-Ozenen \& Sandalli, 2017), while the remaining part was coated with two

layers of acid-resistant nail varnish (Maybelline; Maybelline New York, NY, USA). The

specimens were then embedded in a denture base resin (FEIYING; Yingpai Dental Materials,

Henan, China) and placed in a mold to form cubes of $1 \mathrm{~cm}^{3}(1 \mathrm{~cm} \times 1 \mathrm{~cm} \times 1 \mathrm{~cm})$, with the

buccal enamel surfaces exposed.

\section{Microhardness measurement}

Surface microhardness $(\mathrm{MH})$ values of the specimens were measured using a Vickers

microhardness tester (HV-1000A; Wowei Technology, Beijing, China) with a $200 \mathrm{~g}$ load applied

for $15 \mathrm{~s}$ (Yazkan \& Ermis, 2018). MH of each specimen was measured at the central, left upper,

left lower, right upper, and right lower regions of the exposed enamel window, from which the

mean surface $\mathrm{MH}$ value of each specimen was calculated. Specimens with a baseline $\mathrm{MH}$ value

of 320-370 and with the error within 20 between the five measurement points were selected.

Finally, fifty-six enamel blocks were selected, and twenty-four enamel blocks were excluded.

$\mathrm{MH}$ of each specimen was measured before treatment, after resin infiltration and polishing, and

Peer) reviewing PDF | (2020:10:54743:2:1:NEW 21 Jun 2021) 
119 after artificial demineralization.

120

121

122

123

124

125

126

127

128

129

130

131

132

133

134

135

136

137

138

139

\section{Resin infiltration and polishing}

Randomly select twenty enamel blocks from the included fifty-six enamel blocks and evenly divided into experimental and control groups. In the experimental group, the enamel blocks were treated with resin infiltrant (Icon; DMG Chemisch-Pharmazeutische Fabrik, Hamburg, Germany) (which contained Icon-Etch, Icon-Dry and Icon-Infiltrant). Etching with Icon-Etch for $30 \mathrm{~s}$ was followed by $30 \mathrm{~s}$ of rinsing and $20 \mathrm{~s}$ of drying. Icon-Dry was then applied for $30 \mathrm{~s}$, followed by air-drying to maintain the dryness. When applying Icon-Dry, discoloration in the opaque white areas should be markedly reduced, otherwise the etching and drying processes must be repeated (a maximum of two times). Icon-infiltrant was then applied for $3 \mathrm{~min}$, followed by removal of excess resin and $40 \mathrm{~s}$ of light curing. Icon-infiltrant was then reapplied and allowed to soak for 1 min, followed by removal of excess resin and $40 \mathrm{~s}$ of light curing. Then, the surfaces of specimens were polished using a Rainbow polishing system (SHOFU; Shofu, Kyoto, Japan) using the black, purple, green, and red polishing discs in sequence for $15 \mathrm{~s}$ each at $10000-12$ $000 \mathrm{rpm}$ and a pressure of $0.3-0.6 \mathrm{~N}$. The polished specimens are re-applied with double-layer of acid-resistant nail varnish. Specimens in the control group were not treated with resin infiltrant, but prepared as enamel blocks.

\section{Artificial demineralization}

An artificial demineralization solution was prepared (Zhao \& Gao, 2014) consisting of 2.2 $\mathrm{mmol} / \mathrm{l} \mathrm{Ca}\left(\mathrm{NO}_{3}\right)_{2}, 2.2 \mathrm{mmol} / 1 \mathrm{KH}_{2} \mathrm{PO}_{4}, 50 \mathrm{mmol} / 1 \mathrm{CH}_{3} \mathrm{COOH}, 5.0 \mathrm{mmol} / 1 \mathrm{NaN}_{3}$, and 0.01 $\mathrm{mmol} / \mathrm{l} \mathrm{NaF}$. The final $\mathrm{pH}$ was adjusted to 4.5 with $\mathrm{NaOH}$. Specimens were immersed in a 
beaker (SHUNIU; Shubo, Sichuan, China) containing the demineralizing solution and artificially demineralized for $96 \mathrm{~h}$. The proportion of demineralizing solution per area of exposed enamel window was $2 \mathrm{ml} / \mathrm{mm}^{2}$ (Rocha et al., 2011). The beaker was then sealed and placed in a constant-temperature shaker (BIOBASE; Biobase biological, Shandong, China) at $37^{\circ} \mathrm{C}(57$

$144 \mathrm{rpm} / \mathrm{min})$. The $\mathrm{pH}$ value of the demineralizing solution was checked daily and maintained at $\mathrm{pH}$

4.5. The specimens were artificially demineralized for $96 \mathrm{~h}$ (Huang \& Li, 2012).

Staining and confocal laser scanning microscopy observation

After artificial demineralization, the experimental and control groups were stained with $0.1 \%$

Rhodamine B (Solarbio; Solarbio, Beijing, China) solution for $12 \mathrm{~h}$, allowing the red fluorescent dye to fully mark the pores of the demineralized enamel. Specimens were cut longitudinally at the exposed enamel window area into $1.0 \mathrm{~mm}$-thick slices using a hard-tissue cutting machine. The surfaces were then ground into $300 \mu \mathrm{m}$-thick slices with sandpaper under running water

152 (Huang \& Li, 2012).

\section{Double-fluorescence staining}

In addition, ten enamel blocks were randomly selected from the included fifty-six enamel blocks

for double-fluorescence staining. Etched with Icon-Etch, stained with 0.1\% Rhodamine B ethanol solution for $12 \mathrm{~h}$, then treated with resin infiltrant. After $300 \mu \mathrm{m}$-thick thin slices were sectioned, they were incubated in 30\% hydrogen peroxide (Sanpu; Xi'an Sanpu Chemical Reagent, Xi'an, China) and placed in $37^{\circ} \mathrm{C}$ for $12 \mathrm{~h}$ to bleach the Rhodamine B solution that had not been enclosed by the resin infiltrant. The slices were then immersed in a $50 \%$ ethanol 
161

162

163

164

165

166

167

168

169

170

171

172

173

174

175

176

177

178

179

180

181

and washed with deionized water for $10 \mathrm{~s}$.

The specimens were subsequently observed by confocal laser scanning microscopy (CLSM)

(Leica TCS SP8; Leica Microsystems, Baden-wuerttemberg, Germany) under 400×

magnification. The excitation and emission wavelengths of Rhodamine B were $568 \mathrm{~nm}$ and 590

$\mathrm{nm}$, respectively; those of sodium fluorescein were $488 \mathrm{~nm}$ and $525 \mathrm{~nm}$, respectively. ImageJ

(ImageJ 1.8.0 for Microsoft; National Institutes of Health, Bethesda, USA) software was used to measure the demineralization and penetration depths. The mean value of five measurements was calculated for each specimen.

\section{Simulated toothbrushing}

In addition, the specimens were brushed using an electric toothbrush (Oral-B P3000; Braun OralB/Procter \& Gamble, Schwalbach am Taunus, Germany). Toothpaste slurry was made by mixing $5 \mathrm{~g}$ toothpaste (Cold Acid Ling; Dengkang Oral Care Products, Chongqing, China) with $15 \mathrm{ml}$ artificial saliva (Solarbio; Solarbio, Beijing, China) (main ingredients: deionized water, $\mathrm{NaCl}$, $\mathrm{KCl}, \mathrm{Na}_{2} \mathrm{SO}_{4}, \mathrm{NH}_{4} \mathrm{Cl}, \mathrm{CaCl}_{2} \cdot 2 \mathrm{H}_{2} \mathrm{O}, \mathrm{NaH}_{2} \mathrm{PO}_{4} \cdot 2 \mathrm{H}_{2} \mathrm{O}, \mathrm{CN}_{2} \mathrm{H}_{4} \mathrm{O}, \mathrm{NaF} ; \mathrm{pH}$ : 6.5-7.0) using an electromagnetic stirrer until a homogeneous suspension (slurry) resulted (Kielbassa et al., 2005). The electric toothbrush could oscillate or rotate at a frequency of $7600 \mathrm{rpm}$ and provided a brushing force of $2 \mathrm{~N}$ (standardized vertical loading force) (Zhao et al., 2017; Lee et al., 2019). The simulated brushing time was calculated on the basis of a brushing time of $120 \mathrm{~s}$ twice a day of 28 teeth in the mouth. A tooth has multiple surfaces to be brushed, so the maximum contact time per tooth surface is reported to be $5 \mathrm{~s}$ per day; therefore, the simulated brushing time of 15.2 min for the surfaces of the specimens was evaluated to be equivalent to 1.5 years of tooth 
182

brushing. During the period of simulated toothbrushing, the specimens were placed in artificial saliva overnight (Lee et al., 2019).

\section{Scanning electron microscopy observation}

Twenty-one enamel blocks were selected from the included fifty-six enamel blocks and divide them into seven groups for scanning electron microscopy (SEM) (ZEISS; ZEISS Auriga FIB Crossbeam System, Baden-Wurttemberg, Germany). (The remaining excess enamel block is used to supplement the loss of enamel block during the experiment.) Group (A) was sound enamel blocks that did not treated with resin infiltrant. Group (B) was sound enamel blocks that were treated with resin infiltrant and not polished. Group (C) was sound enamel blocks treated with resin infiltrant following polishing. Group (D) was sound enamel blocks treated with resin infiltrant following 96 hours of artificial demineralization. Group (E) was sound enamel blocks that were not treated with resin infiltrant following 96 hours of artificial demineralization. Group

(F) was sound enamel blocks treated with resin infiltrant following 6 months of simulated toothbrushing. Group $(\mathrm{G})$ was sound enamel blocks that were not treated with resin infiltration following 6 months of simulated toothbrushing. Then, these specimens were ultrasonically cleaned for $5 \mathrm{~min}$, dried to the conventional critical point, and coated using an ion spatter. Surface morphological changes of the specimens were observed under SEM at a magnification of $3000 \times$.

\section{Statistical analysis}

All statistical analyses were performed by SPSS 20.0 statistical software (SPSS 20.0 for Windows; IBM Analytics, Armonk, NY, USA). Data were expressed as means \pm standard 
203

204

205

206

207

208

209

210

211

212

213

214

215

216

217

218

219

220

221

222

223

deviation (SD). Assumption of normal distribution was checked using Kolmogorov-Smirnov

and Shapiro-Wilks tests, and analyzed using one-way analysis of variance (ANOVA). A t-test

was performed to compare the difference between the two groups. Significance levels of $\alpha=0.05$

indicated significant differences.

\section{Results}

\section{Surface microhardness values of specimens in two groups}

As shown in Fig. 2, there was no statistically significant difference in the baseline MH values

between the experimental and control groups $(344.8( \pm 6.0)$ vs. $349.0( \pm 9.2), P=0.240)$,

indicating that there was no significant difference in the degree of initial enamel mineralization

between specimens in the two groups. After resin infiltration, the surface $\mathrm{MH}$ value of samples

in the experimental group was $346.8( \pm 9.7)$, with no significant difference $(P=0.250)$ when

compared with the baseline, indicating that treatment of sound enamel surfaces with resin

infiltrant did not affect the degree of mineralization. After $96 \mathrm{~h}$ of artificial demineralization of

specimens in the two groups, surface MH of specimens in the control group was significantly

decreased compared with that in the experimental group $(266.0( \pm 34.5)$ vs. $304.0( \pm 13.0), P=$

0.017), indicating that the degree of demineralization was higher in the experimental group.

\section{Confocal laser scanning microscopy results}

Red areas in the CLSM images represent the depth of the demineralization in specimens stained

with Rhodamine B and the acid-etched enamel areas sealed by the resin infiltrant; green areas

represent the demineralized microporous areas that were not sealed by resin infiltrant; black

PeerJ reviewing PDF | (2020:10:54743:2:1:NEW 21 Jun 2021) 
224 areas represent air and normal hard tissues that were not stained. The results showed that the

225 demineralization depth in resin-infiltrated specimens (experimental group) was significantly

226 shallower than that in non-resin-infiltrated specimens (control group) $(50.4( \pm 14.3) \mu \mathrm{m}$ vs. 97.9

$227( \pm 22.8) \mu \mathrm{m}, P<0.001$; Fig. 3, Fig. 4). Double-fluorescence staining showed that the resin

228 infiltrant almost completely penetrated the etched demineralized enamel areas, with a mean

229 penetration depth of $31.6( \pm 9.0) \mu \mathrm{m}$ (Fig. 5).

230 Scanning electron microscopy results

231 Changes in enamel surface morphology observed by SEM showed that the surfaces of untreated

232 enamel blocks only had visible scratches (Fig. 6A). After the samples were treated with resin

233 infiltration and not polished, the enamel surfaces showed a dense, rough, fish-scale shaped,

234 enamel prism-like structure (Fig. 6B). After polishing, changes in the surface structure were not

235 obvious and the enamel surfaces became more uniform (Fig. 6C). After $96 \mathrm{~h}$ of artificial

236 demineralization, the surfaces of the untreated enamel blocks exhibited obvious pit-like

237 structures (Fig. 6E) and the enamel prisms of untreated enamel blocks were more severely

238 damaged than those of resin-infiltrated enamel (Fig. 6D). After the simulated toothbrushing

239 abrasion test, the resin-infiltrated enamel blocks showed smooth surfaces with a dense and

240 uniform fish-scale-like structure (Fig. 6F), while the surfaces of the untreated enamel blocks had

241 no obvious special structure and were smooth (Fig. 6G).

Discussion

244 The main goals of modern dentistry are early prevention, early detection, and timely treatment of 
early lesions by promoting remineralization. Resin infiltration is a minimally invasive approach

246 for treating early enamel caries without mechanical destruction of the enamel structure

247 (Kielbassa, Muller \& Gernhardt, 2009; Skucha-Nowak, 2015). To further explore the possibility

248 of using resin infiltrant in the primary prevention of dental caries, in this study, sound permanent

249 enamel surfaces were treated with resin infiltrant followed by further experimental procedures,

250 such as artificial demineralization, simulated toothbrushing, and fluorescent staining to observe

251 changes in the degree of demineralization and surface morphology of the enamel. The results of

252 this study show that the infiltrating resin can improve the ability of a sound enamel surface to

253 resist acid corrosion and demineralization and completely penetrate the acid-etched

254 demineralized enamel area. Accept the null hypothesis.

$255 \mathrm{MH}$ can be used as a parameter to detect changes in the mineral content of dental hard tissue

256 (Gomez et al., 2008); MH changes can reflect mineral loss or gain (Kielbassa et al., 1999). In our

257 study, results from MH measurement showed that preventive application of resin infiltrant has no

258 significant on sound enamel surfaces on its hardness. This finding provides a theoretical basis for

259 further experiment.

260 After artificial demineralization, the degree of demineralization was lower in the experimental group and that resin-infiltrated enamel had strong ability to resist acid erosion and demineralization. The triethylene glycol dimethacrylate (TEGDMA) present in resin infiltrant and hydroxyapatite in enamel constitute a uniform composite, and that the interaction of these crystals improves enamel resistance to acid erosion and slows the loss of mineral content of enamel (Kielbassa et al., 2020). It was previously reported that adding ethanol and TEGDMA to 
266 Icon resin can significantly reduce the viscosity and contact angle of the material, thereby

267 increasing the permeability coefficient. The high permeability of penetrating resin can reduce

268 morbidity, microleakage and secondary dental caries (Chen et al., 2019). However, some studies

269 have shown that the microhardness of infiltrating resin after treatment of early caries cannot be comparable to that of sound enamel (Neres et al., 2017). The penetrating surface did not show complete resistance to new cariogenic challenges (Neres et al., 2017; Torres et al., 2012). In addition, for caries extending into dentin, treatment efficacy of resin infiltration was not significantly different from the non-infiltrated controls (Liang et al., 2018). Therefore, we proposed an experimental design for the treatment of sound enamel surface with resin infiltration. And the results show that infiltrating resin can help sound enamel resist artificial demineralization to a certain extent.

CLSM is a high-resolution microscopy technology that has been widely used in study of dental caries and oral microbiology. In this study, results from CLSM observation after Rhodamine B staining showed that the demineralization depth in the experimental group was less than that in the control group, which confirmed the $\mathrm{MH}$ results. This indicates that resin infiltrant can occlude the micropore structure found in enamel and block the passages that bacteria and acid require to cause further dissolution of the enamel structure. The treated enamel had good mechanical stability that prevented dissolution of the enamel surface structure in an acidic environment, making it more resistant to acid and demineralization. Our result is in line with previous findings by Gurdogan et al. (Gurdogan, Ozdemir-Ozenen \& Sandalli, 2017) that showed that the $\mathrm{MH}$ of resin-infiltrated demineralized enamel increased. Nevertheless, research 
287 has shown that resin infiltration is unable to remineralize the demineralized tooth enamel and to

288 prevent further recurrent caries (Gelani et al., 2014). In addition, Studies have pointed out that

289 with progressed enamel carious lesions the infiltration frequently will be inhomogeneous and

290 incomplete (Schneider et al., 2017). Therefore, the treatment of infiltrating resin for the formed

291 early caries does not guarantee a 100\% success rate. So, we guess that the earlier the use of

292 penetrating resin may have a positive effect on tooth enamel. Our findings further confirm the

293 positive effect of resin infiltrant on improving resistance of sound enamel to acid erosion and

294 demineralization, and provide an experimental basis for the preventive use of resin infiltrant on

295 enamel surfaces.

296

Double fluorescence staining results showed that treatment of sound enamel surfaces with Icon-

297 Etch resulted in slight demineralization of the enamel surface layer, with a mean

demineralization depth of $31.6( \pm 9.0) \mu \mathrm{m}$. This result is consistent with findings from previous

studies investigating the effect of etching gel on enamel surfaces (Meyer-Lueckel, Paris \&

Kielbassa, 2007; Paris, Dörfer \& Meyer-Lueckel, 2010; Neuhaus et al., 2013; Arnold et al.,

2015). Acid etching can increase the surface roughness of the enamel, create microporosity in the

enamel, and ethanol dehydration can increase the penetration ability of low-viscosity resin

(Kielbassa et al., 2005; Ulrich et al., 2015; Yoo et al., 2019; Youssef et al., 2020). In the present

study, staining results showed that resin infiltrant effectively sealed the micropores in sound

enamel formed by an etching gel. Previous studies investigating the effect of resin infiltrant on

caries lesions by Mandava et al. (Mandava et al., 2017) and Liu et al. (Liu et al., 2012) reported 
308

309

310

311

312

313

314

315

316

317

318

319

320

321

322

323

324

325

326

327

328

enamel surfaces. This result also suggests that, for slightly superficial damage to an enamel surface caused by acid etchant, resin infiltrant can completely penetrate demineralized areas.

SEM is based on the interaction of electrons with substances and provides sample images in three dimensions that can reflect the surface structure of the samples. In this study, SEM results showed that sound enamel surfaces showed a scratched appearance after grinding with sandpaper (Fig. 6A). Treatment of sound enamel surfaces with resin infiltrant did not destroy the surface structure of the enamel, and a dense, uniform, but rough, fish-scale-like structure was observed on the enamel surfaces before polishing (Fig. 6B); after polishing, the enamel surfaces were more uniform and smoother (Fig. 6C). Similar results were obtained by Arnold et al. (Arnold, Meyer \& Naumova, 2016) and Mueller et al. (Mueller et al., 2011). Applying resin infiltrant to enamel caries lesions can provide and maintain enamel surfaces. Yazkan's Research proposed that although resin infiltrants are capable of penetrating deeply into the porous enamel lesion, they cannot form a smooth coat on the lesion surfaces (Yazkan \& Ermis, 2018). Rough enamel surfaces increase the chance of bacterial adhesion (Gurdogan, Ozdemir-Ozenen \& Sandalli, 2017) and pigmentation (Arnold, Meyer \& Naumova, 2016), so polishing is indispensable for resin infiltration.

After artificial demineralization, there were a few cracks and shallow pits on the enamel surfaces which treated with resin infiltrant, and changes in the enamel prism structure were not obvious (Fig. 6D); while, obvious pit-like structures were observed on the enamel surfaces which not treated with resin infiltrant and enamel prism structure was markedly destroyed (Fig. 6E). This further confirmed the abovementioned MH and CLSM results. These findings indicated that 
329

330

331

332

333

334

335

336

337

338

339

340

341

342

343

344

345

346

347

348

349

resin infiltrant exerted a significant protective effect on tooth enamel.

After six months of simulated toothbrushing, the enamel surfaces that were not treated with resin infiltrant showed no obvious or clear structure (Fig. 6G). However, compared with the surface morphology of enamel that received no treatment and was not submitted to simulated toothbrushing (Fig. 6A), the enamel surfaces became smooth and the scratches largely disappeared. This may be because abrasive ingredients in toothpaste can cause long-term friction on tooth surfaces under certain pressure of electric toothbrushes, which is similar to the longterm slow effect of polishing. Enamel surfaces treated with resin infiltrant showed a dense and uniform fish-scale-like structure after simulated toothbrushing (Fig. 6F), which showed a smoother surface structure compared with the surface morphology of enamel treated with resin infiltrant that was not subjected to polishing and simulated toothbrushing (Fig. 6B), as well as a clearer surface structure compared with enamel treated with resin infiltrant that was polished, but not subjected to simulated toothbrushing (Fig. 6C). These results indicated that simulated toothbrushing can enable resin-infiltrated enamel surfaces to exhibit a clear, dense, uniform, smooth fish-scale-like structure. We speculate that a certain period of routine toothbrushing simulation can make the enamel surfaces more uniform, smoother, and show certain stability. This further provides a favorable basis for preventive application of resin infiltrant on sound tooth enamel surfaces.

The protective effect of resin infiltrant on sound tooth enamel provides a new attempt on prevention of dental caries, which may be used in preventing dental caries in people at high caries risk, such as patients who will undergo orthodontic treatment. According to reports, the 
350

351

352

353

354

355

356

357

358

359

360

361

362

363

364

365

366

367

368

369

370

incidence of white spot lesions in patients who have not undergone orthodontic treatment is

between 11-24\% (Gulec \& Goymen, 2019). The prevalence of white spot lesions after treatment

fixed orthodontic appliances is $23 \%, 50 \%$ or even $97 \%$ (Kobbe C, et al., 2016). Studies have

shown (Costenoble et al., 2016; Gulec \& Goymen, 2019) that during orthodontic bonding,

bracket bonding performed immediately or shortly following treatment of demineralized enamel

with resin infiltrant did not affect the bonding quality of orthodontic brackets. People at high risk

of caries are more likely to suffer from caries than normal people, which not only affects the

quality of life, but also affects people's physical and mental health. Our study provides a new

possibility for people at high risk of caries.

However, this was an in vitro study, so further studies are needed to confirm the feasibility of

preventive using resin infiltrant to protect sound tooth enamel against erosion and

demineralization. In addition, some studies pointed out resin infiltrant lacks persistent

antibacterial effects and cannot inhibit bacterial growth (Tawakoli \& Attin 2016). Therefore,

some scholars add antibacterial substances, such as silver nanoparticles (AgNP) (Kielbassa et al.,

2020), quaternary ammonium methacrylate (Yu et al., 2020) to provide the effect of the

antibacterial resin penetrant. Our research has not made further discussion on this. Therefore, the

follow-up direction of our research is diverse and worthy of in-depth consideration.

\section{Conclusions}

The findings of this study suggest that resin infiltrant can completely penetrate an acid-etched, demineralized enamel area, effectively seal micropores in the enamel, and improve the ability of

Peer) reviewing PDF | (2020:10:54743:2:1:NEW 21 Jun 2021) 
371 sound enamel surfaces to resist acid erosion and demineralization, making it difficult for external

372 acids to enter gaps present in the enamel. Resin infiltration can therefore play a role in protection

373 of tooth enamel from erosion by acid and demineralization.

374

375 Acknowledgements

376 Conflict of Interest Statement

377 The authors have no conflicts of interest to declare.

378 Author Contributions

379 Meng Li contributed to the data collection, statistical analysis, data interpretation and manuscript

380 preparation; Zhengyan Yang and Yajing Huang contributed to the data collection, statistical

381 analysis, data interpretation and literature search, YueHeng Li and Zhi Zhou contributed to study

382 design, data collection, manuscript preparation and revision. All authors gave final approval and

383 agreed to be accountable for all aspects of the work.

384

Peer] reviewing PDF | (2020:10:54743:2:1:NEW 21 Jun 2021) 
385

386

387

388

389

390

391

392

393

394

395

396

397

398

399

400

401

402

403

404

405

406

407

408

409

410

411

\section{References}

Ammari MM, Soviero VM, da Silva Fidalgo TK, Lenzi M, Ferreira DM, Mattos CT, de Souza IP, Maia LC. 2014. Is non-cavitated proximal lesion sealing an effective method for caries control in primary and permanent teeth? A systematic review and meta-analysis. Journal of Dentistry. 42(10):1217-27. DOI: 10.1016/j.jdent.2014.07.015.

Araújo GS, Naufel FS, Alonso RC, Lima DA, Puppin-Rontani RM. 2015. Influence of Staining Solution and Bleaching on Color Stability of Resin Used for Caries Infiltration. Operative Dentistry. 40(6): E250-6. DOI: 10.2341/14-290-L.

Arnold WH, Haddad B, Schaper K, Hagemann K, Lippold C, Danesh G. 2015. Enamel surface alterations after repeated conditioning with $\mathrm{HCl}$. Head \& Face Medicine. 11:32.

DOI:10.1186/s13005-015-0089-2.

Arnold WH, Meyer AK, Naumova EA. 2016. Surface Roughness of Initial Enamel Caries

Lesions in Human Teeth After Resin Infiltration. Open Dentistry Journal. 10:505-515. DOI:10.2174/1874210601610010505.

Arthur RA, Zenkner JE, d'Ornellas Pereira Júnior JC, Correia RT, Alves LS, Maltz M. 2018.

Proximal carious lesions infiltration-a 3-year follow-up study of a randomized controlled clinical trial. Clinical Oral Investigations. 22(1):469-474. DOI: 10.1007/s00784-017-2135-x.

Askar H, Schwendicke F, Lausch J, Meyer-Lueckel H, Paris S. 2018. Modified resin infiltration of non-, micro- and cavitated proximal caries lesions in vitro. Journal of Dentistry. 74:56-60. DOI: 10.1016/j.jdent.2018.03.010.

Chen M, Li JZ, Zuo QL, Liu C, Jiang H, Du MQ. 2019. Accelerated aging effects on color, microhardness and microstructure of ICON resin infiltration. European Review for Medical and Pharmacological Sciences. 23(18):7722-7731. DOI: 10.26355/eurrev_201909_18981.

Costenoble A, Vennat E, Attal JP, Dursun E. 2016. Bond strength and interfacial morphology of orthodontic brackets bonded to eroded enamel treated with calcium silicate-sodium phosphate salts or resin infiltration. Angle Orthodontist. 86(6):909-916. DOI:10.2319/111315-764.1.

Faghihian R, Shirani M, Tarrahi MJ, Zakizade M. 2019. Efficacy of the Resin Infiltration 
412 Technique in Preventing Initial Caries Progression: A Systematic Review and Meta-Analysis.

413 Pediatric Dentistry. 41(2):88-94.

414 Gelani R, Zandona AF, Lippert F, Kamocka MM, Eckert G. 2014. In vitro progression of

415 artificial white spot lesions sealed with an infiltrant resin. Operative Dentistry. 39(5):481-8. DOI:

416 10.2341/13-202-L.

417 Gurdogan EB, Ozdemir-Ozenen D, Sandalli N. 2017. Evaluation of Surface Roughness

418 Characteristics Using Atomic Force Microscopy and Inspection of Microhardness Following

419 Resin Infiltration with Icon®. Journal of Esthetic \& Restorative Dentistry. 29(3):201-208.

420 DOI:10.1111/jerd.12279.

421 Gomez S, Uribe S, Onetto JE, Emilson CG. 2008. SEM analysis of sealant penetration in

422 posterior approximal enamel carious lesions in vivo. Journal of Adhesive Dentistry. 10(2):151-

423156.

424 Gulec A, Goymen M. 2019. Assessment of the resin infiltration and CPP-ACP applications

425 before orthodontic brackets bonding. Dental Materials Journal. 38(5):854-860.

426 DOI:10.4012/dmj.2019-021.

427 Huang YJ, Li YH. 2012. An in vitro study of remineralization potential of several toothpastes on 428 initial enamel lesions. International Journal of Stomatology. 39(06):710-713.

429 Kashbour W, Gupta P, Worthington HV, Boyers D. 2020. Pit and fissure sealants versus fluoride

430 varnishes for preventing dental decay in the permanent teeth of children and adolescents.

431 Cochrane Database of Systematic Reviews. 11:CD003067. DOI:

432 10.1002/14651858.CD003067.pub5.

433 Kielbassa AM, Wrbas KT, Schulte-Mönting J, Hellwig E. 1999. Correlation of transversal

434 microradiography and microhardness on in situ-induced demineralization in irradiated and

435 nonirradiated human dental enamel. Archives of Oral Biology. 44(3):243-51. DOI:

436 10.1016/s0003-9969(98)00123-x.

437 Kielbassa AM, Gillmann L, Zantner C, Meyer-Lueckel H, Hellwig E, Schulte-Mönting J. 2005.

438 Profilometric and microradiographic studies on the effects of toothpaste and acidic gel abrasivity

Peer] reviewing PDF | (2020:10:54743:2:1:NEW 21 Jun 2021) 
439

440

441

442

443

444

445

446

447

448

449

450

451

452

453

454

455

456

457

458

459

460

461

462

463

464

465

on sound and demineralized bovine dental enamel. Caries Research. 39(5):380-6. DOI:

$10.1159 / 000086844$.

Kielbassa AM, Muller J, Gernhardt CR. 2009. Closing the gap between oral hygiene and minimally invasive dentistry: a review on the resin infiltration technique of incipient (proximal) enamel lesions. Quintessence International. 40(8):663-81.

Kielbassa AM, Leimer MR, Hartmann J, Harm S, Pasztorek M, Ulrich IB. 2020. Ex vivo investigation on internal tunnel approach/internal resin infiltration and external nanosilvermodified resin infiltration of proximal caries exceeding into dentin. PLoS One. 15(1):e0228249. DOI: 10.1371 journal.pone.0228249.

Kobbe C, Fritz U, Wierichs RJ, Meyer-Lueckel H. 2019. Evaluation of the value of re-wetting prior to resin infiltration of post-orthodontic caries lesions. Journal of Dentistry. 91:103243. DOI: 10.1016/j.jdent.2019.103243.

Lasfargues JJ, Bonte E, Guerrieri A, Fezzani L. 2013. Minimal intervention dentistry: part 6. Caries inhibition by resin infiltration. British Dental Journal. 214(2):53-59.

DOI:10.1038/sj.bdj.2013.54.

Lee JH, Kim SH, Han JS, Yeo IL, Yoon HI. 2019. Optical and Surface Properties of Monolithic Zirconia after Simulated Toothbrushing. Materials (Basel, Switzerland). 12(7):1158.

DOI:10.3390/ma12071158.

Liang Y, Deng Z, Dai X, Tian J, Zhao W. 2018. Micro-invasive interventions for managing noncavitated proximal caries of different depths: a systematic review and meta-analysis. Clinical Oral Investigations. 22(8):2675-2684. DOI: 10.1007/s00784-018-2605-9.

Liu Y, Ge L, Chen H, Chi X. 2012. A Study on the Penetration Abilities of Natural Initial Caries Lesions with Resin Infiltration HuaXi KouQiang YiXue ZaZhi. 30(5):483-486.

Liu Chao, Ge Jiuyu, Yin Shuo, Li Wen, Miao Leiying. 2015. Comparison of VAS score and morphology of teeth of elder patients with secondary dentin sensitivity following tooth abrasion before and after penetrating resin treatment. Journal of Jilin Univerity (Medicine Edition). 41(005):1008-1011. DOI:10.13481/j.1671-587x.20150524. 
466 Mandava J, Reddy YS, Kantheti S, Chalasani U, Ravi RC, Borugadda R, Konagala RK. 2017.

467 Microhardness and Penetration of Artificial White Spot Lesions Treated with Resin or Colloidal

468 Silica Infiltration. Journal of Clinical \& Diagnostic Research. 11(4):ZC142-ZC146.

469 DOI:10.7860/JCDR/2017/25512.9706.

470 Markowitz K, Carey K. 2018. Assessing the Appearance and Fluorescence of Resin-Infiltrated

471 White Spot Lesions with Caries Detection Devices. Operative Dentistry. 43(1):E10-E18. DOI:

$472 \quad 10.2341 / 16-153-\mathrm{L}$.

473 Meyer-Lueckel H, Paris S, Kielbassa AM. 2007. Surface layer erosion of natural caries lesions

474 with phosphoric and hydrochloric acid gels in preparation for resin infiltration. Caries Research.

475 41(3):223-230. DOI:10.1159/000099323.

476 Meyer-Lueckel H, Chatzidakis A, Naumann M, Dörfer CE, Paris S. 2011. Influence of

477 application time on penetration of an infiltrant into natural enamel caries. Journal of Dentistry.

478 39(7):465-9. DOI: 10.1016/j.jdent.2011.04.003.

479 Mueller J, Yang F, Neumann K, Kielbassa AM. 2011. Surface tridimensional topography

480 analysis of materials and finishing procedures after resinous infiltration of subsurface bovine

481 enamel lesions. Quintessence International. 42(2):135-147.

482 Neres ÉY, Moda MD, Chiba EK, Briso A, Pessan JP, Fagundes TC. 2017. Microhardness and

483 Roughness of Infiltrated White Spot Lesions Submitted to Different Challenges. Operative

484 Dentistry. 42(4):428-435. DOI: 10.2341/16-144-L.

485 Neuhaus KW, Schlafer S, Lussi A, Nyvad B. 2013. Infiltration of natural caries lesions in

486 relation to their activity status and acid pretreatment in vitro. Caries Research. 47(3):203-210.

487 DOI:10.1159/000345654.

488 Oh HJ, Oh HW, Lee DW, Kim CH, Ahn JY, Kim Y, Shin HB, Kim CY, Park SH, Jeon JG.

489 2017. Chronologic Trends in Studies on Fluoride Mechanisms of Action. Journal of Dental

490 Research. 96(12):1353-1360. DOI: 10.1177/0022034517717680.

491 Oliveira A, Felinto LT, Francisconi-Dos-Rios LF, Moi GP, Nahsan FPS. 2020. Dental

492 Bleaching, Microabrasion, and Resin Infiltration: Case Report of Minimally Invasive Treatment 
493

494

495

496

497

498

499

500

501

502

503

504

505

506

507

508

509

510

511

512

513

514

515

516

517

518

519

of Enamel Hypoplasia. The International Journal of Prosthodontics. 33(1):105-110. DOI:

10.11607/ijp.6232.

Paris S, Dörfer CE, Meyer-Lueckel H. 2010. Surface conditioning of natural enamel caries

lesions in deciduous teeth in preparation for resin infiltration. Journal of Dentistry. 38(1):65-71.

DOI:10.1016/j.jdent.2009.09.001.

Paris S, Meyer-Lueckel H, Kielbassa AM. 2007. Resin infiltration of natural caries lesions.

Journal of Dental Research. 86(7):662-6. DOI: 10.1177/154405910708600715.

Rocha Gomes Torres C, Borges AB, Torres LM, Gomes IS, de Oliveira RS. 2011. Effect of caries infiltration technique and fluoride therapy on the colour masking of white spot lesions. Journal of Dentistry. 39(3):202-207. DOI:10.1016/j.jdent.2010.12.004.

Schneider H, Park KJ, Rueger C, Ziebolz D, Krause F, Haak R. 2017. Imaging resin infiltration into non-cavitated carious lesions by optical coherence tomography. Journal of Dentistry. 60:9498. DOI: 10.1016/j.jdent.2017.03.004.

Schwendicke F, Meyer-Lueckel H, Stolpe M, Dörfer CE, Paris S. 2014. Costs and effectiveness of treatment alternatives for proximal caries lesions. PLoS One. 9(1):e86992. DOI: 10.1371/journal.pone.0086992.

Sekundo C, Frese C. 2020. Underlying Resin Infiltration and Direct Composite Veneers for the Treatment of Severe White Color Alterations of the Enamel: Case Report and 13-Month FollowUp. Operative Dentistry. 2020 Jan/Feb;45(1):10-18. DOI: 10.2341/18-242-L.

Silva SN, Reich AM, DeLeon E Jr, Schafer T, Rueggeberg FA, Fortson WM Jr. 2018. Staining potential differences between an infiltrative resin and an esthetic, flowable composite. Journal of Esthetic \& Restorative Dentistry. 30(5):457-463. DOI: 10.1111/jerd.12415.

Skucha-Nowak M. 2015. Attempt to assess the infiltration of enamel made with experimental preparation using a scanning electron microscope. Open medicine (Warsaw, Poland). 10(1):238248. DOI:10.1515/med-2015-0036.

Tawakoli PN, Attin T, Mohn D. 2016. Oral biofilm and caries-infiltrant interactions on enamel. Journal of Dentistry. 48:40-5. DOI: 10.1016/j.jdent.2016.03.006. 
520 Torres CR, Rosa PC, Ferreira NS, Borges AB. 2012. Effect of caries infiltration technique and

521 fluoride therapy on microhardness of enamel carious lesions. Operative Dentistry. 37(4):363-9.

522 DOI: 10.2341/11-070-L.

523 Ulrich I, Mueller J, Wolgin M, Frank W, Kielbassa AM. 2015. Tridimensional surface roughness 524 analysis after resin infiltration of (deproteinized) natural subsurface carious lesions. Clinical Oral 525 Investigations. 19(6):1473-83. DOI: 10.1007/s00784-014-1372-5.

526 Yazkan B, Ermis RB. 2018. Effect of resin infiltration and microabrasion on the microhardness, 527 surface roughness and morphology of incipient carious lesions. Acta Odontologica Scandinavica. 528 76(7):473-481. DOI: 10.1080/00016357.2018.1437217.

529 Yoo HK, Kim SH, Kim SI, Shin YS, Shin SJ, Park JW. 2019. Seven-year Follow-up of Resin 530 Infiltration Treatment on Noncavitated Proximal Caries. Operative Dentistry. 44(1):8-12. 531 DOI:10.2341/17-323-L.

532 Youssef A, Farid M, Zayed M, Lynch E, Alam MK, Kielbassa AM. 2020. Improving oral health: 533 a short-term split-mouth randomized clinical trial revealing the superiority of resin infiltration 534 over remineralization of white spot lesions. Quintessence International. 51(9):696-709. DOI: 535 10.3290/j.qi.a45104.

536 Yu J, Huang X, Zhou X, Han Q, Zhou W, Liang J, Xu HHK, Ren B, Peng X, Weir MD, Li M, 537 Cheng L. 2020. Anti-caries effect of resin infiltrant modified by quaternary ammonium 538 monomers. Journal of Dentistry. 97:103355. DOI: 10.1016/j.jdent.2020.103355.

539 Zhao X, Gao X. 2014. Effect of Resin Infiltration Treatment on the Colour of White Spot 540 Lesions. HuaXi KouQiang YiXue ZaZhi. 32(3):306-309.

541 Zhao X, Pan J, Zhang S, Malmstrom HS, Ren YF. 2017. Effectiveness of resin-based materials 542 against erosive and abrasive enamel wear. Clinical Oral Investigations. 21(1):463-468. DOI: $543 \quad 10.1007 / \mathrm{s} 00784-016-1814-3$. 
Figure 1

\section{Experimental flow chart related to the experimental procedures in this study.}

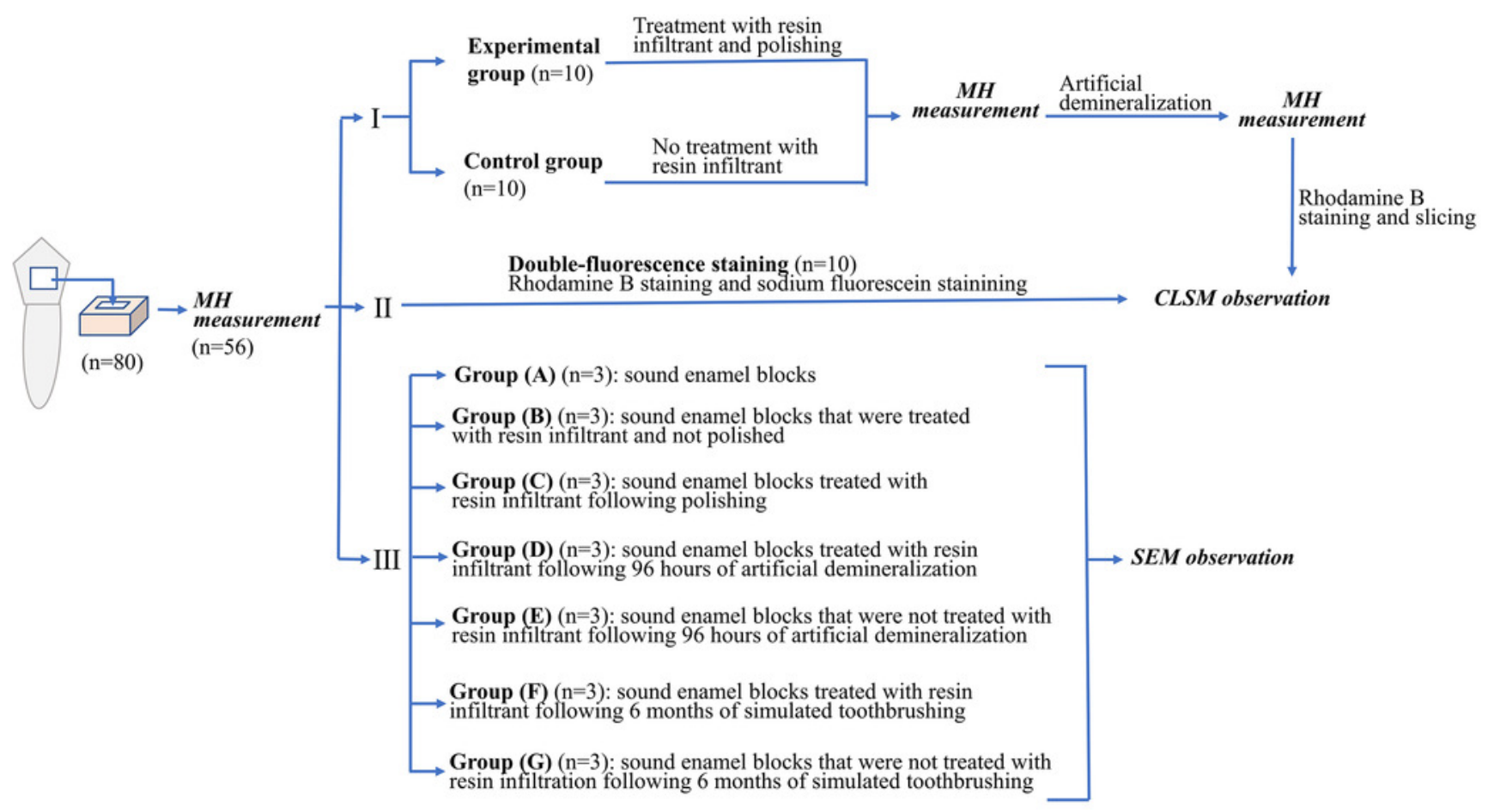


Figure 2

Comparison of surface microhardness values of specimens between control and experimental groups at each time point $(n=10$, mean $\pm S D)$.

$* \mathrm{P}<0.05,{ }^{* * *} \mathrm{P}<0.001, \mathrm{~ns}=$ no significant difference.

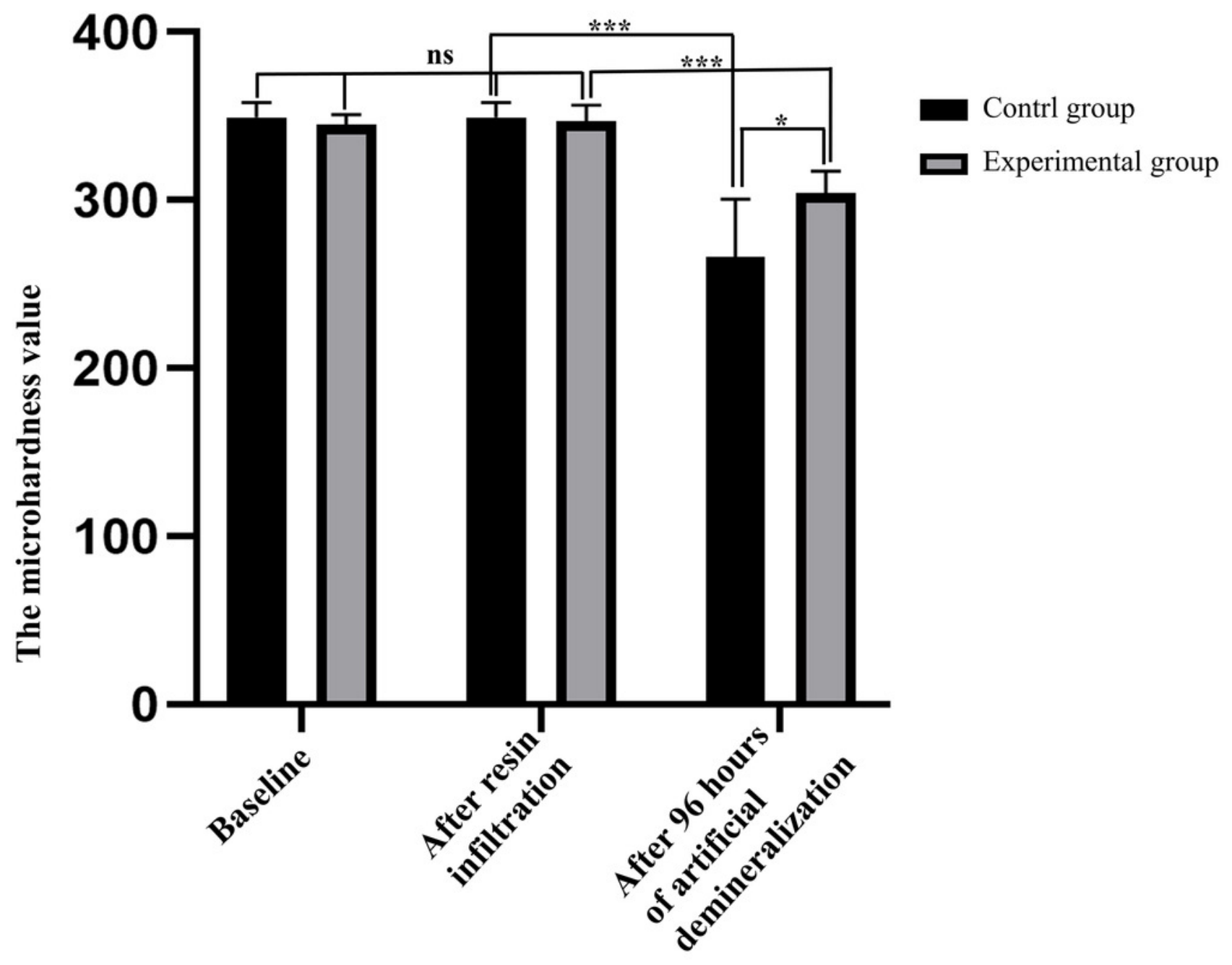


Figure 3

Comparison of demineralization depth of specimens between control and experimental groups $(n=10$, mean $\pm S D)$.

$* * * P<0.001$. 


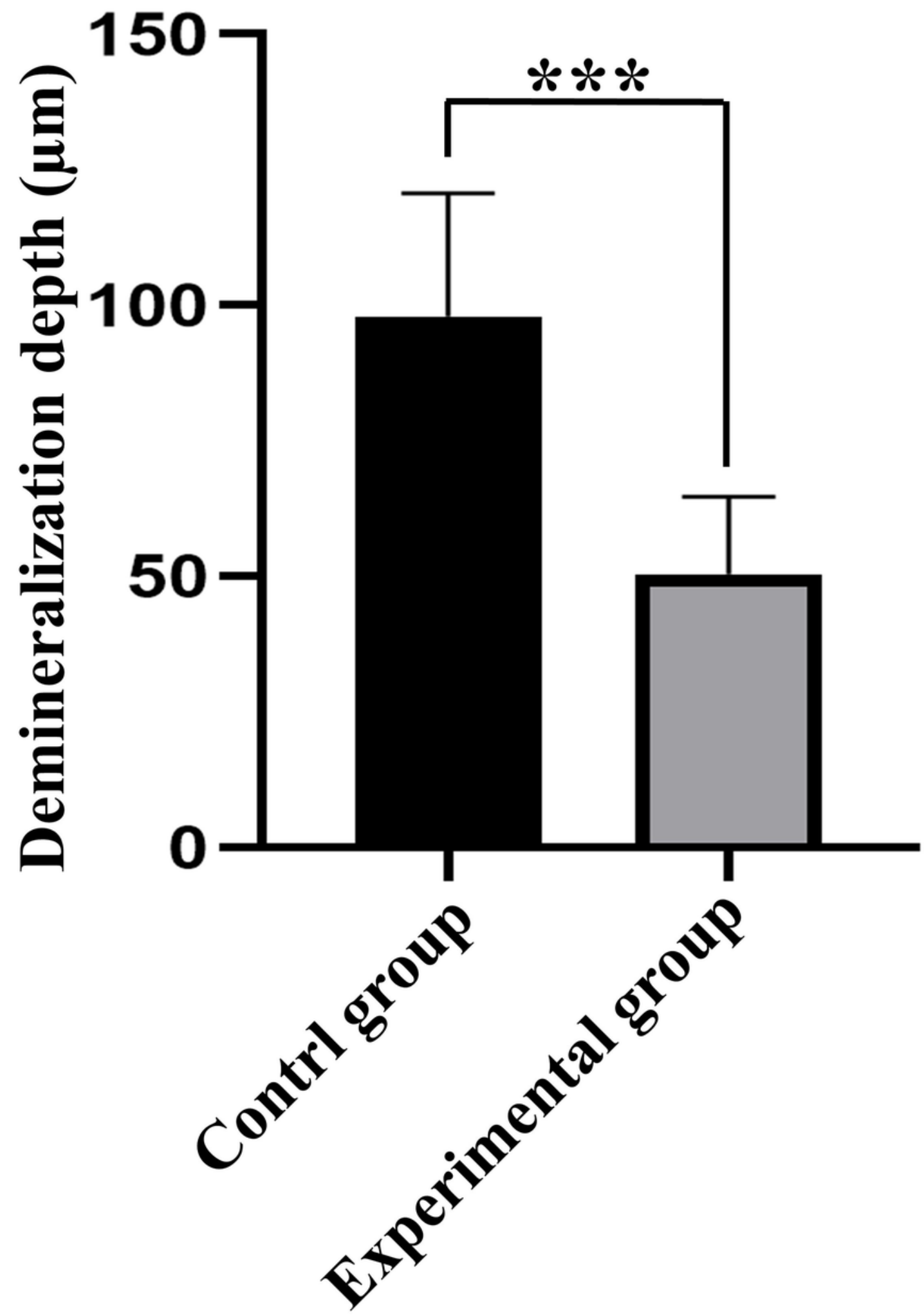




\section{Figure 4}

CLSM images of the specimens after rhodamine B staining, the width of the red area reflects the demineralization depth of enamel.

(A) Staining results of the experimental group specimens after artificial demineralization 96 hours. (B) Staining results of the control group specimens after artificial demineralization 96 hours. Scale bar represents $25 \mu \mathrm{m}$.
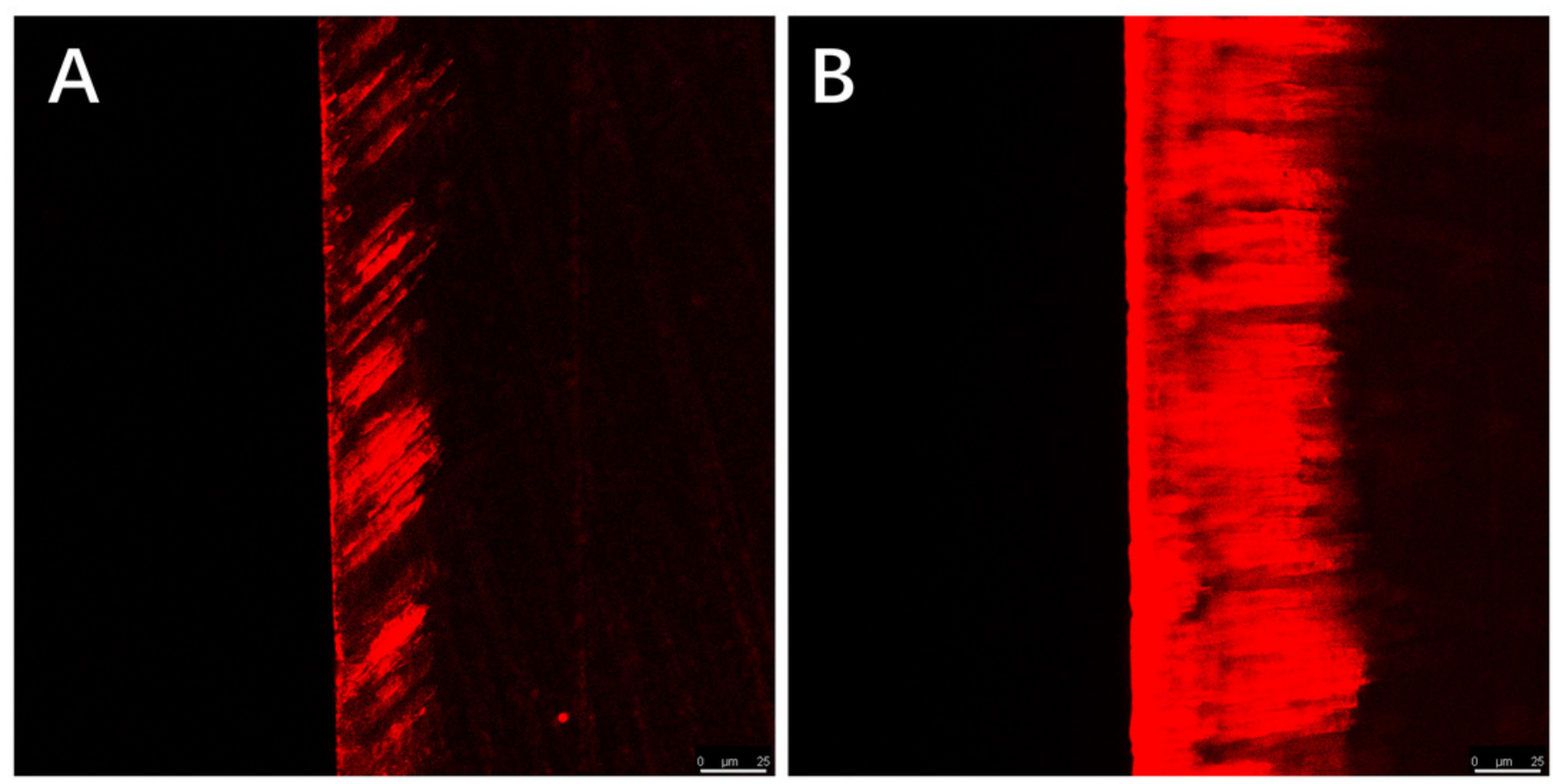


\section{Figure 5}

CLSM images of the specimens after double-fluorescence staining.

(A) Rhodamine B staining results of the specimens after etched with Icon-Etch; red area represents the depth of the acid etched demineralized enamel areas sealed by resin infiltrant. (B) Sodium fluorescein staining results of the specimens after treated with resin infiltrant, sectioned and bleached; green area represents the demineralized microporous areas that are not sealed by resin infiltrant. (C) Merge (A) with (B); shows that resin infiltrant almost completely penetrated into the etched demineralized enamel areas. Scale bar represents $25 \mu \mathrm{m}$.
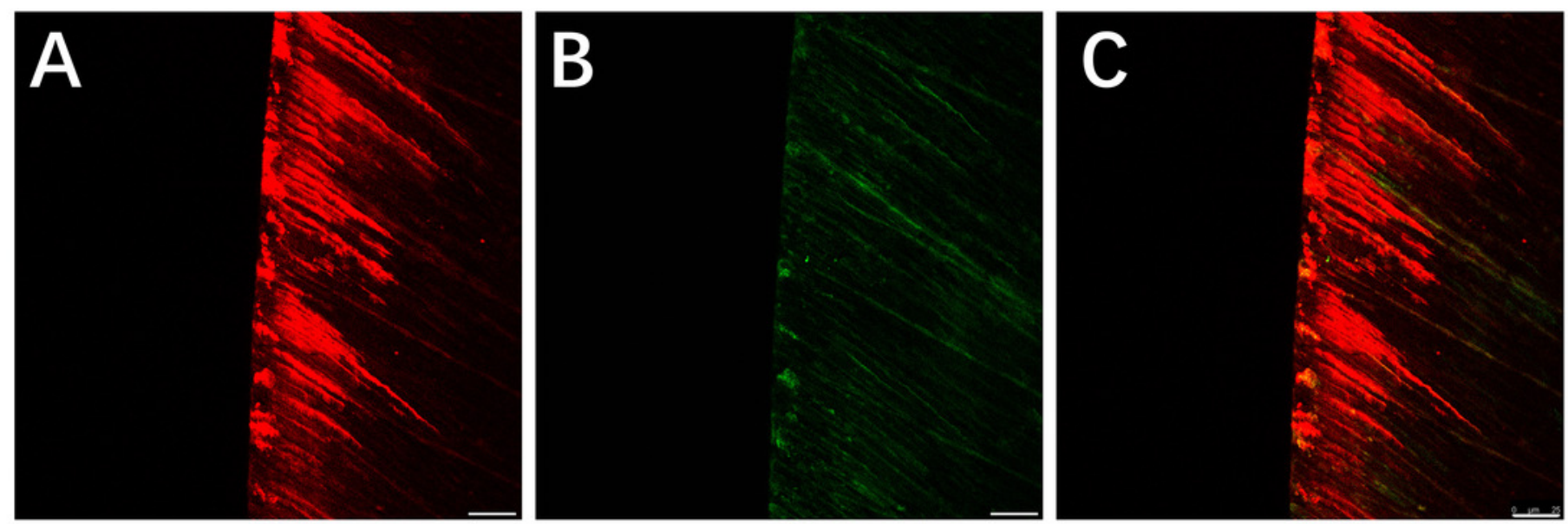


\section{Figure 6}

SEM resutls of surface morphology.

(A) Sound enamel blocks that did not treated with resin infiltrant. (B) Sound enamel blocks that were treated with resin infiltrant and not polished. (C) Sound enamel blocks treated with resin infiltrant following polishing. (D) Sound enamel blocks treated with resin infiltrant following 96 hours of artificial demineralization. (E) Sound enamel blocks that were not treated with resin infiltrant following 96 hours of artificial demineralization. (F) Sound enamel blocks treated with resin infiltrant following 6 months of simulated toothbrushing. (G) Sound enamel blocks that were not treated with resin infiltration following 6 months of simulated toothbrushing.
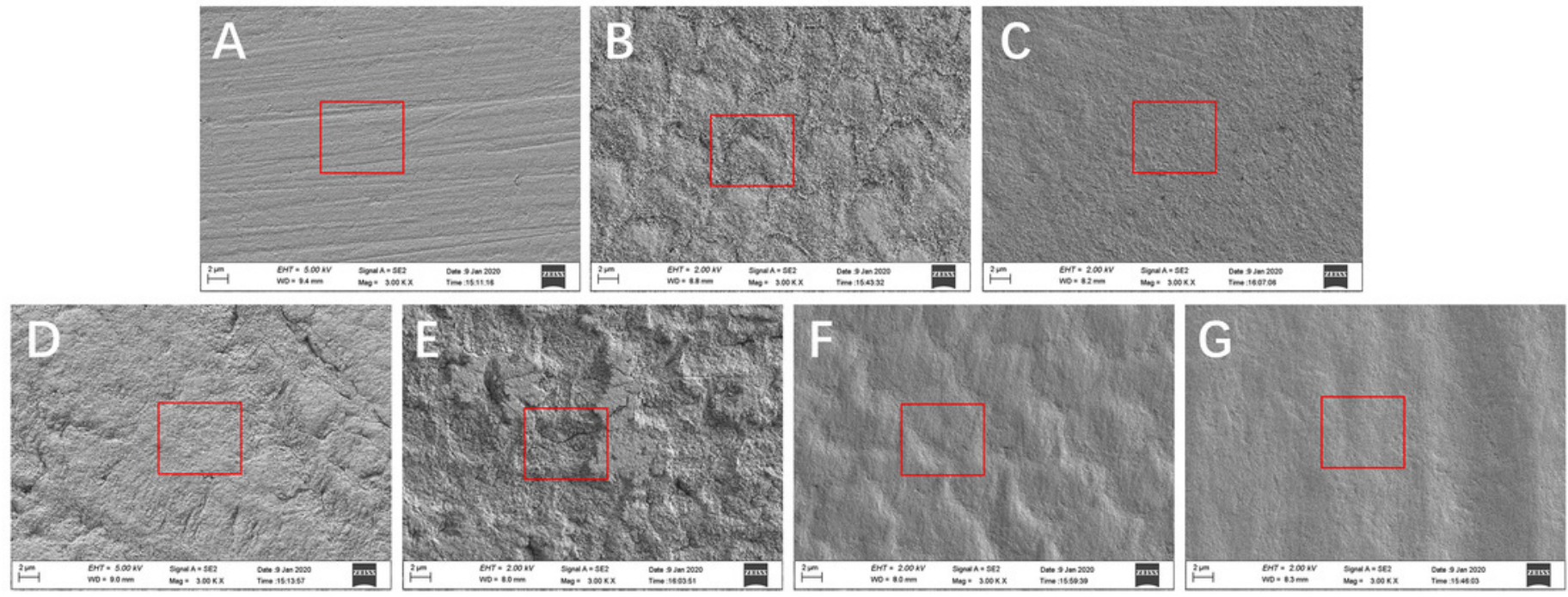